\title{
Enterprise Information Management Performance Evaluation Based on Grey Fuzzy Theory \\ Hui-ying Gao ${ }^{a}$ Xin-bao Liu ${ }^{b}$
}

The School of Management of Hefei University of Technology ,Hefei china

agaohuiyg@sina,com,blxinbao@126.com

\author{
Keywords: Enterprise Information Management; Performance Evaluation; Indicator System; Grey \\ Fuzzy Theory; $\alpha$-Cut Set.
}

\begin{abstract}
Performance evaluation of information management is a necessary part in information management. Regarding information as a kind of resource, this paper constitutes an indicator system framework for enterprise information management performance evaluation, based on effect evaluation principle in performance evaluation. It proposes enterprise information management performance evaluation model based on fuzzy-grey theory and performance level used in calculation of enterprise information management. At last, it makes a sensitivity analysis to evaluation results by $\alpha$-cut set. The effectiveness and rationality of this method can be indicated by example analysis. And sensitivity analysis increases the practical reference value of evaluation results to enterprises.
\end{abstract}

\section{Introduction}

Enterprise information management studies the information management activities of enterprises, it is an important part for enterprise performance evaluation to carry out performance evaluation of enterprise information management. Domestic scholars have made related research on performance evaluation of enterprise information from different views. For example, Cheng Yang ${ }^{[2]}$ established a set of information performance evaluation system which can be dynamically assessed according to implementation phases, after he made an analysis research on the life cycle in the implementation process of enterprise information. Zhang Song ${ }^{[3]}$ classifies enterprise IT resources into IT infrastructure resources, IT human resources and complimentary organizational resources with the basis of RBV. He proposed hierarchical model for information performance evaluation based on RBV. According to three functional orientations in general enterprise information management system and daily operation security, Yang Shubin ${ }^{[4]}$ developed an indicator system for information management system performance evaluation. Tian Shang ${ }^{[5]}$ constituted an indicator system for performance evaluation of enterprise information development, including information management, infrastructure, human resources, operation environment and enterprise revenue, according to the thought of system comprehensive evaluation.

In the above research, assessment is made from the perspective of information technology or information systems. No comprehensive performance evaluation of information is made from the perspective of information management. In terms of enterprise information management, so far, there has not been a complete and scientific performance evaluation system. Most choices for evaluation methods are qualitative analysis methods and quantitative analysis methods are not that much. In performance evaluation of enterprise information management, some factors which influence information management are ambiguous due to some unclear factors and uncertain evaluation criteria. Because some evaluators have different abilities and preferences, evaluation information is often incomplete or inadequate (meaning gray). Therefore, from a management perspective, this paper regards information as a resource. By the optimal allocation of this scarce resource called information, it establishes the indicator system framework for the enterprise information management performance evaluation. Considered the incompleteness of information and uncertainty of concepts in enterprise information management, this paper proposes enterprise information management performance evaluation model based on gray fuzzy theory. Example analysis shows that some validity and application value can be found to assess enterprise information management performance with grey fuzzy evaluation methods. 


\section{Evaluation Indicator Construction for Enterprise Information Management Performance}

Indicator system for performance evaluation of enterprise information management is not only the premise of the performance evaluation of enterprise information management, but also the core to build up the theoretical framework for performance evaluation of the entire enterprise information management. The quality of indicators directly determines the quality of performance evaluation, thus affecting people's judgment towards the enterprise information management performance. When this paper sets indicator system for performance evaluation of enterprise information management, it starts from the content component of the resource allocation in the enterprise information. With the basis of indicator system proposed by the National Information Evaluation Center, it sets up the following 6 dimensions and details to the performance evaluation of enterprise information management, from perspective of reflecting the independence, relativity, normalization and economical efficiency of evaluation indicator:(1)Information background dimension. It is the indicator to reflect the subject configuration of enterprise information resources. At the information age, enterprises must plan enterprise information management strategy from the perspectives of development and overall situation. They should attach great importance to the enterprise information management, so that enterprise information management strategy and corporate development strategies complement with each other to further develop;(2) Human resources dimension. It is the indicator to reflect the main configuration of enterprise information resources, because human resources are the mediators and main users of the information assets; (3) Information development dimension is an indicator for reflection of the ontology configuration of enterprise information resource. It is an essential means to effectively explore and use information in the realization of optimum distribution of information resources. Its basic indicator level refers to the coverage rate of information technology tools in information collection, the building level of enterprise web portals and the effective access to information; (4) Infrastructure dimension. It is the indicator to reflect table body configuration of enterprise information resources. When we emphasize the configuration of the information infrastructure, we can further optimize the configuration structure of information resources. However, the rationality of configuration structure largely determines the effect of information resources configuration; (5) Applications situation dimensions. It reflects enterprise information resources table body configuration as an indicator. It refers to channel resources and technical resources configuration when information resources are transmitted from information ontology resources to information subject resources; (6) Information security dimension. It reflects enterprise information resources table body configuration as an indicator. It refers to the configuration of security resources used in the transmission from information ontology resources to information subject resources.

In summary, Figure 1 shows the establishment of two levels of indicator system for performance evaluation of enterprise information management. 


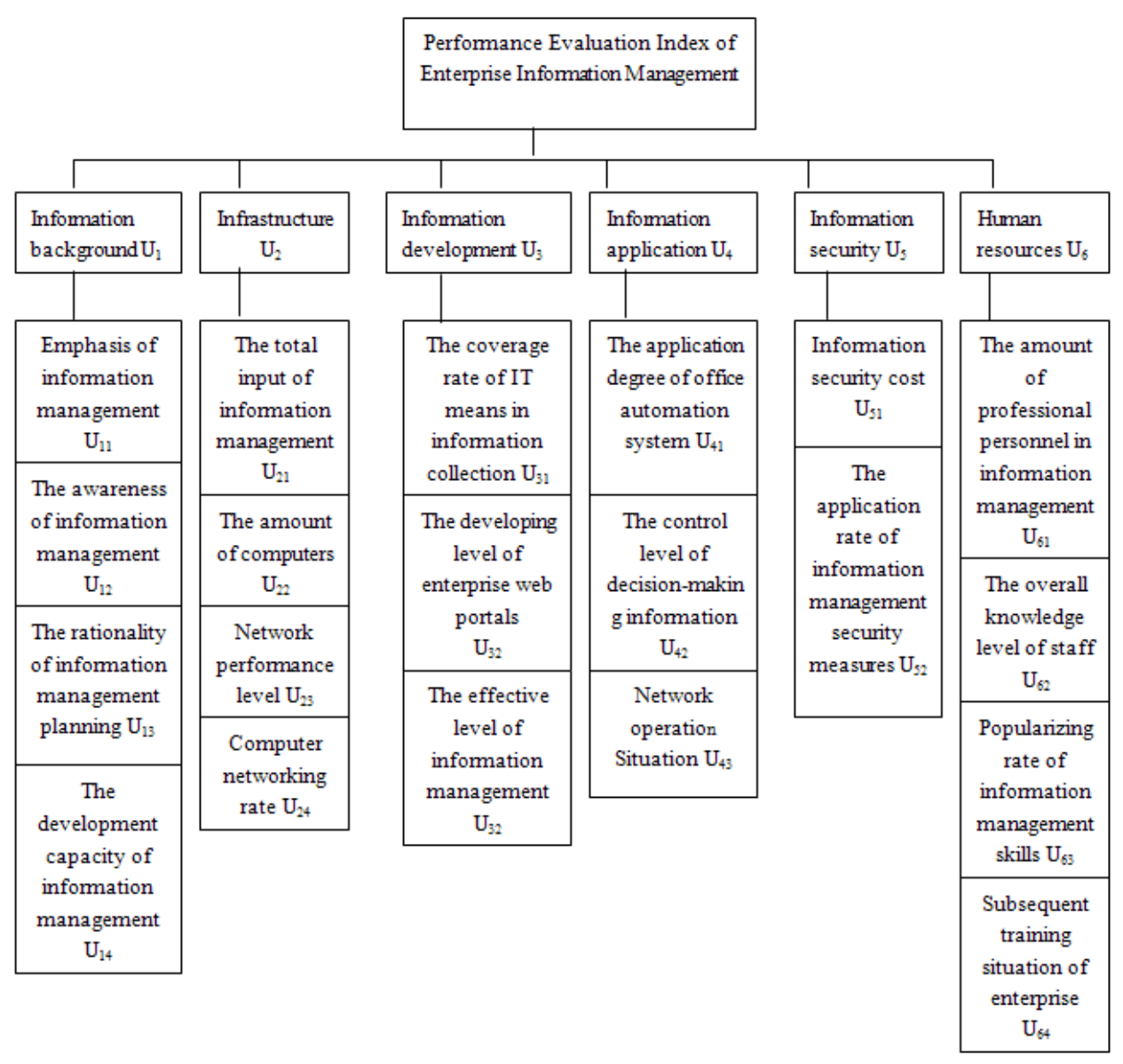

Figure 1 Indicator system for performance evaluation of enterprise information management

\section{-Grey fuzzy comprehensive evaluation model of enterprise information management performance}

In the evaluation practice of enterprise information management performance, fuzziness is always accompanied by grey, that is, there is the undefined fuzziness of concept, as well as the grey for incomplete information that can not be descript by precise math language. The thesis builds a method of comprehensive evaluation based on grey fuzzy theory, which combines the grey theory and fuzzy theory, and aiming at solving the fuzziness and the grey of expert evaluation information in enterprise information management performance evaluation. According to grey fuzzy math theory and common fuzzy evaluation method, the math model and method of grey fuzzy comprehensive evaluation can be given with the following steps:

Analyze influencing factor system. Evaluation factor set is the collection of factors which can influence enterprise information management performance, in which the factors are classified by property, building the hierarchical systems. The sub-factor set of evaluation object is $U=\left\{U_{1}, U_{2}, \cdots, U_{m}\right\}$ (first level indicator), in which every sub-factor can be divided into the next stage subset, meaning the second stage factor $\operatorname{set} U_{i}=\left\{U_{i 1}, U_{i 2}, \cdots, U_{i k}\right\}$ (second level indicator), $i=1,2, \cdots, m, k$ is the factor in $U_{i}$, see Chart 1 .

Build comment set. The comment set is the collection of various evaluation results. If the comment set is $v=\left\{v_{1}, v_{2}, \cdots, v_{n}\right\}$, it is denoted as $\mathrm{v}=$ Excellent, good, medium, poor $\}$. Because the amount of information can not be measured by number, the thesis uses some descriptive words to correspond with certain grey range, for example, the information is divided into the following classes:\{ very sufficient, relatively sufficient, medium, relatively poor, very poor\}, corresponding with certain grey value $\{0 \sim 0.2,0.2 \sim 0.4,0.4 \sim 0.6,0.6 \sim 0.8,0.8 \sim 1.0\}$. 
Determine weight set. The weight set is used to descript the grey fuzzy relation between evaluation object and indicator set, giving the weight and relevant grey scale of every factor in the same level about the above level norm to form the weight set: $\underset{\otimes}{\widetilde{A}}=\left[\left(a_{1}, v_{1}\right),\left(a_{2}, v_{2}\right), \cdots,\left(a_{m}, v_{m}\right)\right]$. Among them, $a_{i}$ is called as the fuzzy part (FP in short) of $\underset{\otimes}{\widetilde{A}}$, and $v_{i}$ is called as the grey part (GP in short) of $\underset{\otimes}{\widetilde{A}}$. Every weight value satisfies the requirement of normalization, meaning

$$
\sum_{i=1}^{m} a_{i}=1
$$

Build evaluation matrix. Evaluation matrix can be considered as the grey fuzzy relation between factor set and comment set. According to certain factor, the author gives the degree of membership of evaluation object to every factor in comment set, and the relevant grey level in condition of information sufficiency. Suppose the evaluation matrix:

$$
\underset{\otimes}{\widetilde{R}}=\left[\begin{array}{cccc}
\left(\mu_{11}, v_{11}\right) & \left(\mu_{12}, v_{12}\right) & \ldots & \left(\mu_{1 n}, v_{1 n}\right) \\
\left(\mu_{21}, v_{21}\right) & \left(\mu_{22}, v_{22}\right) & \ldots & \left(\mu_{2 n}, v_{2 n}\right) \\
\ldots & \ldots & \ldots & \ldots \\
\left(\mu_{m 1}, v_{m 1}\right) & \left(\mu_{m 2}, v_{m 2}\right) & \ldots & \left(\mu_{m n}, v_{m n}\right)
\end{array}\right]
$$

Conduct complex evaluation. To save more evaluation information, the author uses $\mathrm{M}(\bullet, \oplus)$ operator in FP, and $(\bigwedge, \oplus)$ operator in GP, so the result of grey fuzzy comprehensive evaluation is:

$$
\underset{\otimes}{\widetilde{B}}=(\underset{\otimes}{\widetilde{A}}, \underset{\otimes}{\widetilde{R}})=\left[\left(b_{j}, v_{b j}\right)\right]_{n}=\left[\left\{\left(\sum_{k=1}^{m} a_{k} \cdot \mu_{k j}\right), \prod_{k=1}^{m}\left(1 \wedge\left(v_{k}+v_{k j}\right)\right)\right\}\right]_{n}
$$

\section{Evaluation result management}

(1) Convert into triangular fuzzy number

Because of the insufficiency and incredibility of grey descriptive information, the amount of information can not be measured by number, and the grey fuzzy value has to be converted into triangular fuzzy number. The set formed by the triangular fuzzy number converted from evaluation result can be marked as: $\underset{\otimes}{\widetilde{B}}=\left[b_{j}\left(1-v_{b j}\right), b_{j}, b_{j}\left(1+v_{b j}\right)\right]$

(2) Convert triangular fuzzy number into interval number by cut set $\alpha$

Suppose under cut set $\alpha$, the minimum of triangular fuzzy number $\omega_{i}=\left(\omega_{i}^{l}, \omega_{i}^{m}, \omega_{i}^{u}\right)$ is $\omega_{i}^{l_{k}}$, and $\operatorname{maximum}$ is $\omega_{i}^{u_{k}}, \omega_{i}^{l_{k}}=\underset{x \in X}{\operatorname{Inf}}\left\{x \mid \mu_{M}(x) \geq \alpha_{k}\right\}, \omega_{i}^{u_{k}}=\underset{x \in X}{\operatorname{Sup}}\left\{x \mid \mu_{M}(x) \geq \alpha_{k}\right\}$ see Chart 2:

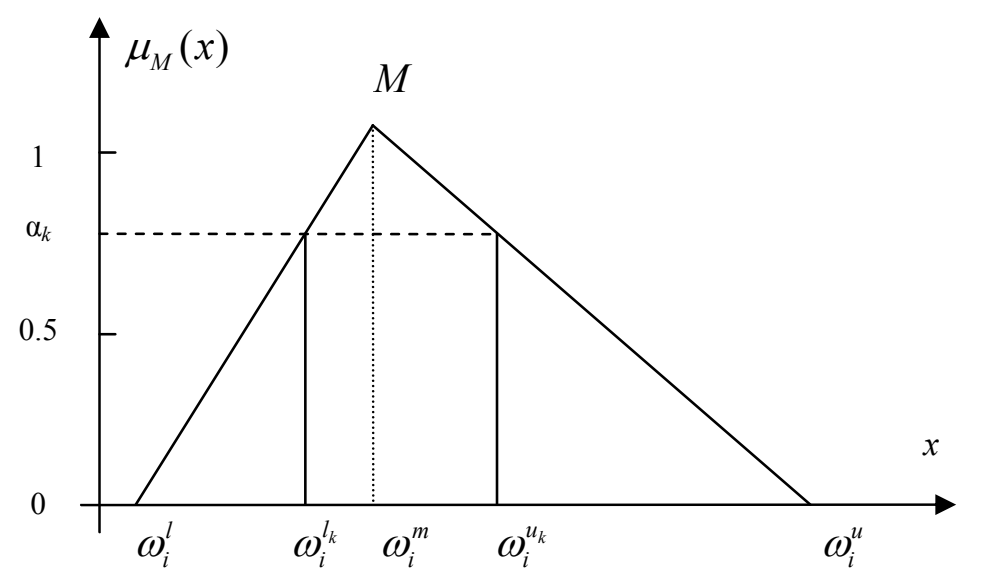

Chart 2 Minimum and maximum of triangular fuzzy number $\omega_{i}$ under cut set $\alpha$

$$
\omega_{i}^{l_{k}}=\omega_{i}^{l}+\alpha_{k}\left(\omega_{i}^{m}-\omega_{i}^{l}\right)
$$




$$
\omega_{i}^{u_{k}}=\omega_{i}^{u}-\alpha_{k}\left(\omega_{i}^{u}-\omega_{i}^{m}\right)
$$

From the above formula, we can see when the value of $\alpha_{k} \quad\left(0<\alpha_{k} \leq 1\right)$ is given, the interval which enterprise information manager vest to evaluation result by credit degree greater than or equal to $\alpha_{k}$ probability can be certain, meaning $\left[\omega_{i}^{l_{k}}, \omega_{i}^{u_{k}}\right]$.

(3) Make interval number in sequence

The method of making interval number in sequence is as follows:

Suppose there are two intervals $\tilde{a}$ and $\widetilde{b}, \widetilde{a}=\left[a^{L}, a^{U}\right], \widetilde{b}=\left[b^{L}, b^{U}\right]$, notes $l_{\widetilde{a}}=a^{U}-a^{L}$, $l_{\widetilde{b}}=b^{U}-b^{L}$, marking as

$$
p(\tilde{a} \geq \tilde{b})=\frac{\min \left\{l_{\widetilde{a}}+l_{\tilde{b}}, \max \left(a^{U}-b^{L}, 0\right)\right\}}{l_{\widetilde{a}}+l_{\widetilde{b}}}
$$

is the possibility degree of $\widetilde{a} \geq \widetilde{b}, \widetilde{a}, \widetilde{b}$, whose sequence relation is $\underset{a}{ } \geq \widetilde{b}$.

To compare interval numbers of the given group in pairs $i=1,2, \cdots, n$ can get the relevant possibility degree $p\left(\widetilde{a}_{i} \geq \widetilde{b}_{j}\right)$, denoted as $p_{i j}(i=1,2, \cdots, n ; j=1,2, \cdots, n)$, and the matrix is $p=\left(p_{i j}\right)_{n \times n}$ called as possibility degree matrix.

The possibility degree matrix $\mathrm{P}$ is fuzzy complementary judgment matrix. Its ordering vector $d=\left(d_{1}, d_{2}, \cdots, d_{n}\right)$ can be calculated from the following formula:

$$
d_{i}=\frac{1}{n(n-1)}\left(\sum_{j=1}^{n} p_{i j}+\frac{n}{2}-1\right), \quad i=1,2, \cdots, n
$$

By comparing every component in ordering vector $d=\left(d_{1}, d_{2}, \cdots, d_{n}\right)$ of possibility degree matrix $\mathrm{P}$, we can get the sequence of interval numbers, as well as the conclusion of corresponding enterprise information management performance evaluation

(4) Conduct sensitivity analysis according to different $\alpha_{\text {value }}$

From chart 2, we can see different ${ }^{\alpha_{k}}$ produces different interval parameter, so it's necessary to make sensitivity analysis for different $\alpha_{k}$ to get reasonable and feasible evaluation result. The value of $\alpha_{k}$ should be analyzed specifically according to the actual condition of enterprise information management.

\section{Example analysis}

A comprehensive evaluation of an enterprise information management will be conducted at present, and many experts are invited to conduct on-site scoring.

Evaluation factors set is shown in Figure 1, and the common set indicates the size of the enterprise information management performance for the $\mathrm{v}=$ \{excellent, good, medium, poor . Analytic Hierarchy Process (Analytic Hierarchy Process) is applied to determine each factor at the same level concerning the weight and corresponding grayscale on the previous level to constitute weight set, where the weight values ought to be normalized. Table 1 shows the weight set of evaluation factors and evaluation matrix by expert scoring, in which A gray and B gray indicate 0.4 and 0.3 respectively. 
Table 1 Weight and grey scale of each factor

\begin{tabular}{|c|c|c|c|c|c|c|c|}
\hline \multirow{2}{*}{ First Grade Indicator } & \multirow{2}{*}{ Second Grade Indicator } & \multirow{2}{*}{ Weight } & \multicolumn{4}{|c|}{ Grade layer } & \multirow{2}{*}{$\begin{array}{l}\text { Gray } \\
\text { scale }\end{array}$} \\
\hline & & & Excellent & Good & Medium & Poor & \\
\hline \multirow{4}{*}{$\begin{array}{l}\text { Information Background } \\
(0.13)\end{array}$} & $\begin{array}{l}\text { Degree of information } \\
\text { management attention }\end{array}$ & 0.43 & 0.1 & 0.5 & 0.2 & \multirow{2}{*}{$\begin{array}{l}0.1 \\
0.2\end{array}$} & 0.3 \\
\hline & $\begin{array}{l}\text { Awareness level of information } \\
\text { management }\end{array}$ & 0.22 & 0.1 & 0.3 & 0.4 & & 0.6 \\
\hline & $\begin{array}{l}\text { Rationality of information } \\
\text { management planning }\end{array}$ & 0.17 & 0.2 & 0.3 & 0.3 & 0.2 & 0.4 \\
\hline & $\begin{array}{lr}\text { Development capacity } & \text { of } \\
\text { information management }\end{array}$ & 0.28 & 0.3 & 0.5 & 0.1 & 0.1 & 0.4 \\
\hline \multirow{4}{*}{ Infrastructure $(0.23)$} & $\begin{array}{l}\text { The proportion of information } \\
\text { management investment }\end{array}$ & 0.2 & 0.1 & 0.6 & 0.3 & 0.1 & 0.4 \\
\hline & $\begin{array}{l}\text { Computer ownership } \\
\text { per hundred }\end{array}$ & 0.29 & 0.2 & 0.4 & 0.3 & 0.1 & 0.2 \\
\hline & Level of network performance & 0.19 & 0.5 & 0.2 & 0.1 & 0.2 & 0.4 \\
\hline & Computer networking rate & 0.32 & 0.2 & 0.5 & 0.3 & 0.1 & 0.2 \\
\hline \multirow{3}{*}{ Application Status (0.15) } & $\begin{array}{l}\text { Applications degree of office } \\
\text { automation system }\end{array}$ & 0.53 & 0.1 & 0.4 & 0.3 & 0.2 & 0.4 \\
\hline & $\begin{array}{l}\text { The level of decision-making } \\
\text { information management }\end{array}$ & 0.15 & 0.3 & 0.2 & 0.4 & 0.1 & 0.6 \\
\hline & Network operation status & 0.32 & 0.2 & 0.5 & 0.2 & 0.1 & 0.3 \\
\hline \multirow{2}{*}{ Information Security $(0.17)$} & $\begin{array}{l}\text { Cost ratio used in information } \\
\text { security }\end{array}$ & 0.61 & 0.4 & 0.3 & 0.2 & 0.1 & 0.3 \\
\hline & $\begin{array}{l}\text { Application rate of information } \\
\text { management security measures }\end{array}$ & 0.39 & 0.2 & 0.4 & 0.2 & 0.2 & 0.4 \\
\hline \multirow{3}{*}{ Information Development $(0.11)$} & $\begin{array}{l}\text { Information technology tools } \\
\begin{array}{l}\text { coverage } \\
\text { collection }\end{array}\end{array}$ & 0.31 & 0.2 & 0.3 & 0.5 & 0 & 0.4 \\
\hline & $\begin{array}{l}\text { Level of Enterprise } \\
\text { building }\end{array}$ & 0.46 & 0.5 & 0.3 & 0.1 & 0.1 & 0.3 \\
\hline & $\begin{array}{l}\text { The effective level of } \\
\text { information management }\end{array}$ & 0.23 & 0.2 & 0.4 & 0.3 & 0.1 & 0.8 \\
\hline \multirow{4}{*}{ Human Resources (0.21) } & $\begin{array}{l}\text { Ownership of information } \\
\text { management expertise }\end{array}$ & 0.23 & 0.2 & 0.3 & 0.4 & 0.1 & 0.2 \\
\hline & Staff overall level of knowledge & 0.37 & 0.4 & 0.2 & 0.2 & 0.2 & 0.2 \\
\hline & $\begin{array}{l}\text { Popularizing rate of information } \\
\text { management skills }\end{array}$ & 0.28 & 0.2 & 0.4 & 0.3 & 0.1 & 0.4 \\
\hline & $\begin{array}{l}\text { Follow-up training } \\
\text { enterprises }\end{array}$ & 0.12 & 0.1 & 0.4 & 0.4 & 0.1 & 0.6 \\
\hline
\end{tabular}

Concluded by gray fuzzy algorithm:

$$
\begin{aligned}
\widetilde{B}_{1}= & \widetilde{A}_{\otimes} \circ \widetilde{R}_{1}=[(0.43,0.3),(0.22,0.3),(0.17,0.3),(0.28,0.3)] \circ \\
& {\left[\begin{array}{llll}
(0.1,0.3) & (0.5,0.3) & (0.2,0.3) & (0.1,0.3) \\
(0.1,0.6) & (0.3,0.6) & (0.4,0.6) & (0.2,0.6) \\
(0.2,0.4) & (0.3,0.4) & (0.3,0.4) & (0.2,0.4) \\
(0.3,0.4) & (0.5,0.4) & (0.1,0.4) & (0.1,0.4)
\end{array}\right] } \\
= & {[(0.183,0.2646),(0.472,0.2646),(0.253,0.2646),(0.149,0.2646)] }
\end{aligned}
$$


Similarly, it is available to

$$
\begin{aligned}
\underset{\otimes}{\widetilde{B}}=\underset{\otimes}{\vec{A}} \underset{\otimes}{\widetilde{R}}= & {[(0.13,0.4),(0.23,0.4),(0.15,0.4),(0.17,0.4),(0.11,0.4),(0.21,0.4)] \circ } \\
& {\left[\begin{array}{cccc}
(0.83,0.2646) & (0.472,0.2664) & (0.253,0.2664) & (0.149,0.2664) \\
(0.237,0.1225) & (0.434,0.1225) & (0.262,0.1225) & (0.119,0.1225) \\
(0.162,0.378) & (0.402,0.378) & (0.283,0.378) & (0.153,0.378) \\
(0.322,0.42) & (0.339,0.42) & (0.2,0.42) & (0.139,0.42) \\
(0.338,0.42) & (0.323,0.42) & (0.27,0.42) & (0.069,0.42) \\
(0.262,0.1575) & (0.303,0.1575) & (0.298,0.1575) & (0.137,0.1575)
\end{array}\right] } \\
= & {[(0.249,0.10),(0.378,0.10),(0.261,0.10),(0.126,0.10)] }
\end{aligned}
$$

Transforming the evaluation result into a collection of triangular fuzzy numbers[0.22,0.249, $0.27],[0.33,0.378,0.41],[0.24,0.261,0.29],[0.12,0.126,0.14]$.

Suppose $\alpha=0.9$, using the cut set to transform triangular fuzzy number into interval number. According to the equation (1) and (2), the interval number is calculated to be: [0.2461, 0.2511], [0.3732, 0.3812], [0.2589, 0.2639], [0.1254, 0.1274].

Sort the interval numbers, and the possibility degree matrix of the sequence according to equation (3) is: $P=\left(\begin{array}{cccc}- & 0 & 0 & 1 \\ 1 & - & 1 & 1 \\ 1 & 0 & - & 1 \\ 0 & 0 & 0 & -\end{array}\right)$

According to the equation (4), the ordering vector is $(0.167,0.3333,0.25,0.0833)$. That the second component is the largest. Therefore, the performance of an enterprise information management is good.

The enterprise principle can vest the credit interval with evaluation result by credit degree greater than or equal to $\alpha$ probability according to actual situation of the enterprise information management and personal knowledge background. Conducting simulation calculation as $\alpha=0.7$, $0.5,0.3$, the obtained ordering vector and assessment results are as shown in Table 2.Assessment results in Table 2:

Table 2 Comparing of assessment results under four $\alpha$ cut sets

\begin{tabular}{|l|l|l|}
\hline$\alpha$ Value & Ordering Vector & Assessment Result \\
\hline 0.9 & $(0.167,0.3333,0.25,0.0833)$ & Good \\
\hline 0.7 & $(0.168,0.3333,0.165,0.167)$ & Good \\
\hline 0.5 & $(0.182,0.3333,0.235,0.083)$ & Good \\
\hline 0.3 & $(0.187,0.3333,0.229,0.083)$ & Good \\
\hline
\end{tabular}

In above four different $\alpha$ values, the performance evaluation rating of the enterprise information management is good. Therefore, this assessment method not only effectively combines the views of the experts groups with different knowledge backgrounds, but also takes into account the actual situation of the enterprise information management. And the sensitivity analysis increases actual reference value of assessment results to the enterprise.

\section{-Conclusions}

In the situation of lacking effective quantitative research in assessing the performance of enterprise information management, this paper establishes a two-tier indicator system for enterprise information management performance evaluation through the optimal allocation of corporate information resources from the perspective of information management. This article characterizes the relationship between the factors and the performance evaluation rating by fuzzy relation, and 
describes the inadequate extent of the information in grayscale, presenting enterprise information management performance evaluation model based on grey fuzzy theory. With the $\alpha$ cut set method, it conducts the sensitivity analysis for assessment results, resulting in comparable quantitative results. It is effective and reasonable to apply this method to the performance evaluation of the enterprise information management. It not only gains the performance evaluation rating of enterprise information management, but also reflects its relative credibility, and a scientific basis is provided to self-diagnosis and adjustment for the enterprise information management performance. Therefore, the grey fuzzy comprehensive evaluation has good practical value in the performance evaluation of enterprise information management.

Foundation Item: Anhui University research base project of humanities and social science: Based on the evidence of the listed company's financial performance evaluation method research

Author: Hui-ying Gao: Female, Suzhou, Anhui, $\mathrm{PhD}$, lecturer, research direction: multiple attribute decision making, assessment methods; Xin-bao Liu :Male, Anqing, Anhui, Ph.D., professor, research direction: group decision-making, assessment methods

\section{References}

[1] Sifeng LIU. Grey System Theory and Its Application .Wuhan, Science Press, 2008 pp. 74-93.

[2] Yang CHENG, Jie ZHANG, Zhaorong QU. Enterprise Information Performance Evaluation System and Its Assessment Method ,Computer Engineering.2007 (2)270-273.

[3] Song Zhang, Xuyong WU. The Evaluation Level Model of Enterprise Information Technology Performance Based on RMV ,Science of Science and Technology Management. 2007 (6)129-133.

[4] Shubin YANG. Fuzzy AHP-Based Information Management System Performance Evaluation ,Business Times. 2011 (21) 90-92.

[5] Shang TIAN, Chun LI. Evaluation Indicator System Construction of Enterprise Information Construction Performance, Statistics and Decision.2010 (14) 175-176.

[6] Bernardin, HJ, Hangan, CM, Kane, J.S. \& Villanova, P., In Performance Appraisal,State of the Art in Practice, Simither, J. Weditor, San Francisco,Jossey-Bass Publishers, 1998. 